\title{
Solvation Dynamics at Aqueous Lipid-Membrane Interfaces Explored by Temperature-Dependent 3-Pulse-Echo Peak Shifts: Influence of the Lipid Polymorphism
}

\author{
Helge Bürsing, ${ }^{\dagger}$ Santi Kundu, ${ }^{\dagger}$ and Peter Vöhringer $*, \dagger, \uparrow$ \\ Biomolecular and Chemical Dynamics Group, Max-Planck-Institute for Biophysical Chemistry, \\ Am Fassberg 11, 3700 Göttingen, Germany, and Université Louis Pasteur, Institut Le Bel, \\ 4 rue Blaise Pascal, F-67000 Strasbourg, France
}

Received: September 20, 2002; In Final Form: January 7, 2003

\begin{abstract}
Three-pulse photon-echo peak-shift (3PEPS) experiments were performed to investigate structural relaxations relevant to solvation dynamics of a cyanine dye which is noncovalently anchored to aqueous interfaces of unilamellar phospholipid vesicles. For comparison, complementary 3PEPS measurements were performed on the same optical chromophore dissolved in bulk water. The influence of the lipid polymorphism is studied in great detail through the temperature dependence of the 3PEPS decay. As opposed to the main order/ disorder phase transition connected with packing phenomena in the hydrophobic membrane core, the rippled gel-to-gel pretransition strongly modifies the solvation response on time scales below 2 ps. This finding indicates that the pretransition is connected with structural modifications of the hydration shell surrounding the polar headgroups of the lipids and is consistent with a dehydration of the interface upon cooling the rippled-gel phases and entering the pure gel phases of the membranes. A comparison between the temperature dependencies of the 3PEPS decay and the linear absorption spectrum strongly suggests that a formal division of water above lipid membranes into "bound" and "free" species only is too simplistic.
\end{abstract}

\section{Introduction}

Solvation phenomena at aqueous phospholipid membrane interfaces are of tremendous importance for charge and mass transport across biological boundaries either by passive permeation (diffusion) or mediated by integral membrane-bound transport proteins and ion channels. ${ }^{1-3}$ This is simply because the dielectric medium surrounding the biological membrane defines the surface potential and thus the work function required for transferring a unit charge ion across the water-membrane interface. More importantly, the dynamics of charge transport processes are strongly influenced by the temporal structural response through which a condensed phase environment seeks to accommodate a dynamically evolving charge distribution. ${ }^{4-6}$ Prominent examples for charge transport phenomena in biological environments are light-driven electron and proton-transfer processes such as those involved in photosynthesis and bacterial ATP production.

The structural reorganizations relevant to dynamic solvation can be studied experimentally by ultrafast switching of the charge distribution of an optical chromophore immersed in the condensed phase system. The switching can be accomplished through an electronically resonant interaction of the chromophore with an ultrashort optical pulse. The subsequent relaxation of the solvation free energy can then be quantified by recording the spectro-temporal evolution of the chromophore's emission usually referred to as the time-dependent fluorescence Stokes shift (TDFSS). ${ }^{7-9}$

\footnotetext{
* To whom correspondence should be addressed. Phone: +49 (551) 201 1333. Fax: +49 (551) 201 1341. E-mail: pvoehri@gwdg.de.

Max-Planck-Institute for Biophysical Chemistry.

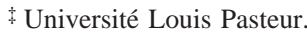

Using such an approach, solvent relaxation in phospholipid bilayers following electronic excitation of membrane-bound optical chromophores has previously been studied on nanosecond time scales by reconstructing their dynamic emission spectra from time-correlated single-photon counting data. ${ }^{10-13}$ Similarly, solvation dynamics of probe molecules confined to the inner water pool of reversed micelles ${ }^{14,15}$ and phospholipid vesicles ${ }^{16-18}$ have also been studied. However, despite its particular relevance for biological transport phenomena, a comprehensive understanding of the ultrafast dynamics of interface-specific solvation in lipid membranes is still lacking mostly because such dynamics have neither been fully addressed by unambiguous experiments nor by means of computer simulations.

The difficulties in performing an unambiguous experiment on dynamic interfacial solvation arise primarily from an inherent roughness of the membrane surface which, in turn, is responsible for a spatially rather ill-defined transition between the inner hydrophobic core of the phospholipid bilayer and the aqueous bulk phase located at an unknown distance above the hydrophilic phospholipid headgroups. ${ }^{19,20}$ The resultant structural heterogeneity in this region is evidenced by molecular dynamics simulations on aqueous phospholipid membranes, which reveal a complex distribution (and polarization) of water along the membrane normal and pronounced water penetration into the hydrophobic-hydrophilic boundary of the bilayer. ${ }^{21-27}$ Furthermore, the dielectric constant across this structurally highly inhomogeneous assembly varies in less than $3 \mathrm{~nm}$ from about 2 (in the hydrocarbon interior) to roughly 80 which is characteristic for bulk water. ${ }^{28}$ Hence, an accurate knowledge of the location of the optical chromophore in the bilayer is imperative if quantitative conclusions are to be drawn from measurements on dynamic solvation in membranes. In addition, 
complementary experiments on the same optical chromophore in aqueous bulk solutions are required to facilitate a clear distinction of interfacial from bulk contributions to the ensemble solvation response.

The dynamics of aqueous bulk solvation has previously been studied in TDFSS experiments by Barbara and co-workers. ${ }^{29}$ Using a water soluble Coumarin derivative, the observed solvation correlation function, $C(t)$, was well modeled by a biexponential decay with time constants of 160 fs and 1.2 ps. Similarly, Jimenez et al. ${ }^{30}$ also obtained a biexponential decay of $C(t)$ for Coumarin 343 in water whose time constants were 55 and 880 fs. The ultrafast portion of the solvation dynamics was attributed to the librational solvent response. For comparison with these experiments, Marcus and co-workers calculated the solvation correlation function in liquid water for spherically and ellipsoidally shaped chromophores dressed with a given dipole density. ${ }^{31}$ As solvent-specific input, they employed the full frequency-dependent dielectric function of the pure liquid. ${ }^{32}$ The long-time tail of their $C(t)$ was found to decay exponentially with a time constant of 810 fs. They concluded that this tail contains contributions which arise from the response of intermolecular modes of the liquid with higher frequencies, i.e., the restricted translational degrees of freedom connected with bending and stretching of hydrogen bonds formed between nearest-neighbor molecules in the liquid. ${ }^{32}$

Recently, we reported on solvation dynamics of a cyanine dye dissolved in liquid water as probed by 3-pulse photon-echo peak-shift (3PEPS) measurements. ${ }^{33}$ The basic conclusion drawn from these studies were fully in line with the computer simulations of Marcus and co-workers. The dominant part of the 3PEPS data decayed on a time scale of roughly $1 \mathrm{ps}$ and was assigned to the solvation response brought about by the restricted translational degrees of freedom of the liquid while a very small component on a time scale of a few picoseconds was tentatively attributed to single molecule rotational diffusion of water. In parallel, we studied the dynamics of solvation for the very same cyanine chromophore when it is noncovalently attached to the aqueous interface of unilamellar phospholipid vesicles. ${ }^{33}$ Anchoring of the optical chromophore was achieved by means of long-chain alkyl residues, which penetrate deeply into the hydrophobic core of the membrane bilayer. The very high specificity of the optical chromophore to the polar membrane interface was evidenced by high-resolution confocal microscopy. ${ }^{34}$ The 3PEPS data obtained at the membrane interface indicated that, already on subpicosecond time scales, solvation of the cyanine chromophore is considerably perturbed in comparison to the bulk. This finding was taken as a signature of a substantial disruption of the bulk water hydrogen-bonded network that is induced by the presence of the polar phospholipid headgroups. ${ }^{33}$

Here, we present complementary 3PEPS studies to elucidate in detail the temperature dependence of biointerface-specific solvation dynamics. Phospholipid membranes exhibit a rich polymorphism that can be traced back primarily to ordering phenomena within the hydrophobic core. ${ }^{3,35}$ The structural modifications associated with the complex thermodynamic phase behavior of lipid membranes has been the subject of numerous experimental studies ranging from X-ray and neutron scattering to nuclear magnetic resonance and vibrational spectroscopy. ${ }^{19,20}$ However, to our knowledge, the present study is the first attempt at exploring possible interrelations between the polymorphism of lipid membranes and ultrafast structural relaxations occurring specifically at their interface to the aqueous surroundings.

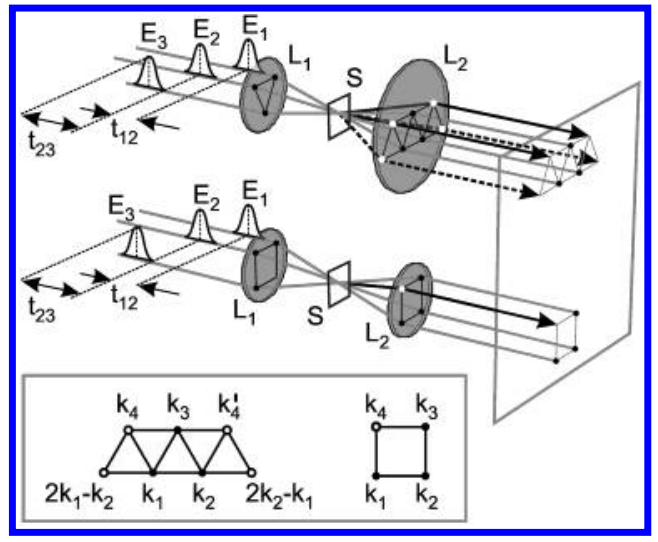

Figure 1. Photon-echo setup in the triangular (top) and boxcar (middle) geometries and view onto the plane of the detector (bottom). $E_{i}=$ optical pulses with wavevectors, $k_{i}(i=1,2$, and 3$)$, and photon echoes $k_{4}$ and $k_{4}^{\prime} . \mathrm{L}_{i}=$ lenses, $\mathrm{S}=$ sample, $t_{12}=$ coherence period, $t_{23}=$ waiting period.

\section{Experimental Section}

Absorption spectra were measured with a UV-vis spectrophotometer (Shimadzu-UV160). Likewise, emission spectra were recorded with a fluorescence spectrometer (Fluorolog2, Perkin-Elmer) equipped with a Kr-arc lamp and a monochromator for excitation. Specific heat measurements were performed with a differential scanning calorimeter (Microcal, LLC) at a scan rate of $5^{\circ} \mathrm{K} / \mathrm{h}$.

The 3PEPS experiments were carried out with a home-built noncollinearly phase-matched and white light-seeded Type-IBBO optical parametric amplifier (NOPA) which was pumped by $150-\mathrm{fs}, 80-\mu \mathrm{J}$ pulses from a frequency-doubled Ti:sapphire regenerative amplifier (RGA). At a repetition rate of $1 \mathrm{kHz}$, the NOPA provided pulses centered around $650 \mathrm{~nm}$ with durations as short as $20 \mathrm{fs}$ and energies as high as $8 \mu \mathrm{J}$. The NOPA output was attenuated to approximately $10 \mathrm{~nJ}$ and was subsequently (pre)compensated for group-velocity dispersion of the NOPA optics as well as that of the photon-echo setup by a pair of fused silica prisms. A set of three identical Al-coated $50 \%$-beam-splitter cubes was used to prepare a sequence of three identical pulses whose timing was adjusted by computercontrolled translation stages (Melles Griot, Nanomover). Photonechoes were generated in both boxcar and triangular phasematching geometries (see Figure 1) as previously described ${ }^{36,37}$ using plano-convex singlets with a focal length of $350 \mathrm{~mm}$ and a bisecting angle of 15 mrad between each pair of pulses. To avoid local heating, the samples were contained in thermostatic optical cells, which were excentrically rotated at $50 \mathrm{rpm}$ around an axis perpendicular to the cell windows. This frequency guaranteed a fresh sample volume for each laser shot. The sample temperature was controlled with an accuracy of $\pm 1{ }^{\circ} \mathrm{C}$.

All lipids were purchased from Avanti Polar Lipids and were used without further purification. Multilamellar vesicles (MLV) of dimyristoyl- and dipalmitoyl-phosphatidylcholine (DMPC and DPPC, Figure 2A) in water were prepared and labeled with the chromophore, 1, 1' -dioctadecyl-3,3,3',3'-tetramethyl-indodicarbocyanine chloride (C18-DiD, Figure $2 \mathrm{~B}$ and $\mathrm{C}$ ), according to ref 38 to provide samples with a molecular dye-to-lipid ratio below $10^{-3}$ at a lipid concentration of $20 \mathrm{mM}$. The labeled MLVs were subsequently extruded through 100 -nm pore poly carbonate filters (Avestin) to obtain unilamellar vesicles with diameters around $100 \mathrm{~nm}$ as shown previously by electron microscopy. ${ }^{39}$ For comparison with dynamics in bulk water, the water soluble but otherwise identical chromophore, 1, 1',3,3, 3', $3^{\prime}$ - 


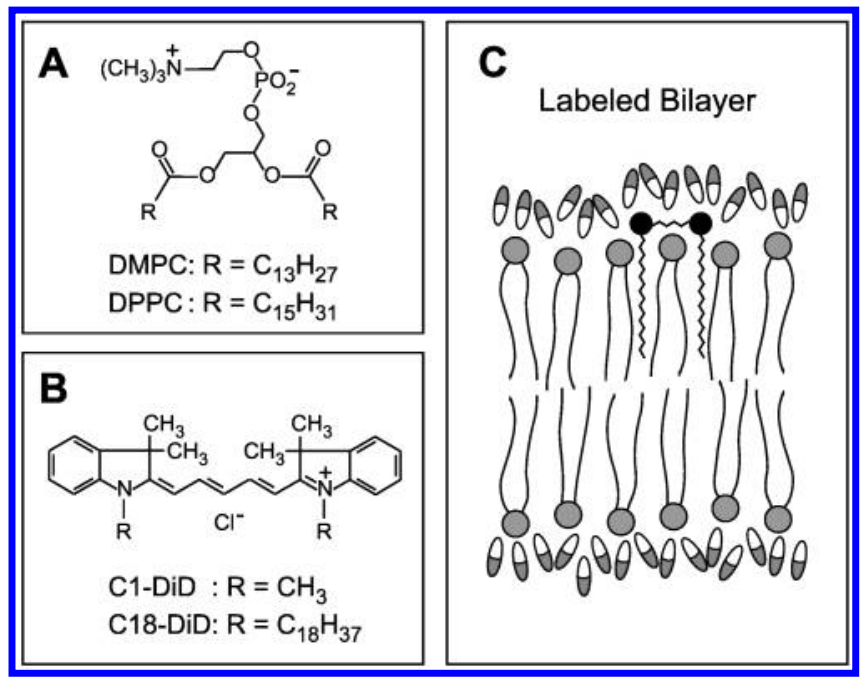

Figure 2. Chemical constitution of the phospholipids (A) and the optical chromophores (B). Sketch of the orientation of C18-DiD at the membrane interface $(C)$.

hexamethyl-indodicarbocyanine chloride, (C1-DiD, Figure 1B) was also studied.

\section{Background}

The dynamics of solvation of an optical chromophore immersed in a condensed phase environment can be described by the normalized energy gap correlation function, $C(t)$

$$
C(t)=\frac{\Delta E(t)-\Delta E(t=\infty)}{\Delta E(t=0)-\Delta E(t=\infty)}
$$

where $\Delta E(t)$ is the time-dependent mean energy gap between the electronic ground and excited states of the chromophore after its optical excitation. In the limit where the electronic excitation presents a small perturbation to the condensed phase system, $C(t)$ is identical to the correlation function

$$
M(t)=\frac{\left\langle\delta \omega_{\mathrm{ge}}(0) \delta \omega_{\mathrm{ge}}(\mathrm{t})\right\rangle}{\left\langle\delta \omega_{\mathrm{ge}}{ }^{2}\right\rangle}
$$

of equilibrium fluctuations, $\delta \omega_{\text {ge }}$, of the optical transition frequency of the chromophore. These fluctuations give rise to line broadening of the linear electronic resonance and are caused by structural relaxations of the microenvironments surrounding the chromophore at thermal equilibrium. ${ }^{40}$ The denominator in eq 2 represents the mean squared amplitude of the energy gap fluctuations or equivalently, the strength, $\Delta^{2}$, with which structural relaxations are coupled to the optical transition.

As shown by Wiersma, Fleming and co-workers, ${ }^{41-43}$ a good estimate for the decay of $M(t)$ can be obtained from 3PEPS measurements. Here, the chromophore interacts with a sequence of three identical ultrashort pulses which are tuned preferentially to its $0-0$ transition. The time-integrated third-order polarization (i.e., the 3-pulse echo signal), is recorded as a function of both interpulse delays, $t_{12}$ and $t_{23}$ :

$$
I_{3 \mathrm{PE}}\left(t_{12}, t_{23}\right)=\int_{-\infty}^{\infty}\left|P^{(3)}\left(t_{12}, t_{23}, t\right)\right| \mathrm{d} t
$$

During the former, the chromophore resides in an optical coherence whereas during the latter, it is found in a (ground or excited state) population. Hence, $t_{12}$ and $t_{23}$ are denoted the coherence and population (or waiting) periods, respectively. In a 3PEPS experiment, the maximum of the 2-dimensional data set, $I_{3 \mathrm{PE}}\left(t_{12}, t_{23}\right)$, along the coherence period is determined as a function of the waiting time. The corresponding coherence delay of maximal echo intensity is termed echo peak shift, $S\left(t_{23}\right)$.

In the Bloch limit, the magnitude of this peak shift is a measure of the ratio between inhomogeneous and homogeneous contributions to the electronic line broadening dynamics. ${ }^{36}$ However, in condensed systems, a separation between homogeneous and inhomogeneous broadening is invalid because of the numerous overlapping time scales on which structural relaxations occur. ${ }^{41-44}$ Thus, with increasing waiting delay, the transition frequency fluctuates on a growing manifold of time scales until for infinite $t_{23}$, and it can finally sample the full spectral width of the linear ensemble resonance. This corresponds to an increasing homogeneity with increasing $t_{23}$ as the chromophore reports the dynamically growing configuration space of its environment via its own transition frequency. As a result, the 3PEPS decays with increasing $t_{23}$. It was shown that, under certain boundary conditions, $S\left(t_{23}\right)$ mirrors the decay of $M(t)$ exactly except for the earliest times where free-induction decay (FID) and/or vibrational dephasing phenomena result in extraneous contributions that are unrelated to environmentspecific dynamics. ${ }^{36,45,46}$

To simulate the nonlinear polarization, $P^{(3)}\left(t_{12}, t_{23}, t\right)$, a perturbative description of four-wave mixing is usually employed. ${ }^{40}$ The central quantity in modeling the dynamics of the condensed phase system is the so-called line broadening function, $g(t)$, which can be computed from $M(t)$ according to ${ }^{40}$

$$
\begin{aligned}
g(t)= & \int_{0}^{t} \mathrm{~d} \tau_{1} \int_{0}^{\tau_{1}} \mathrm{~d} \tau_{2} M\left(\tau_{2}\right)= \\
& \Delta^{2} \int_{0}^{t} \mathrm{~d} \tau_{1} \int_{0}^{\tau_{1}} \mathrm{~d} \tau_{2} M^{\prime}\left(\tau_{2}\right)-i \lambda \int_{0}^{t} \mathrm{~d} \tau\left[1-M^{\prime \prime}(\tau)\right]
\end{aligned}
$$

The auxiliary functions, $M^{\prime}(t)$ and $M^{\prime \prime}(t)$, on the rhs of eq 4 are related to the real and imaginary parts of $M(t)=\operatorname{Re}[M(t)]+i$ $\operatorname{Im}[M(t)]$ through the relations

$M^{\prime}(t)=\frac{1}{\Delta^{2}} \operatorname{Re}[M(t)]$ and $M^{\prime \prime}(t)=1+\frac{1}{\lambda} \int_{0}^{\mathrm{t}} \mathrm{d} \tau \operatorname{Im}[\mathrm{M}(\tau)]$

Proper normalization requires the coupling strength, $\Delta^{2}$, and the reorganization energy, $\lambda$, to become

$$
\Delta^{2}=\operatorname{Re}[M(0)] \text { and } \lambda=-\int_{0}^{\mathrm{t}} \mathrm{d} \tau \operatorname{Im}[M(\tau)]
$$

Because the real and imaginary parts of $M(t)$ are entangled by the fluctuation-dissipation theorem, the auxiliary functions are related by a cosine transformation followed by an inverse cosine transformation $^{40}$

$$
\begin{array}{r}
M^{\prime}(t)=\frac{\lambda}{\pi \Delta^{2}} \int_{0}^{\infty} \mathrm{d} \omega \cos (\omega \mathrm{t}) \omega \operatorname{coth}\left(\hbar \omega / 2 k_{\mathrm{B}} T\right) \times \\
\int_{0}^{\infty} \mathrm{d} \tau \cos (\omega \tau) M^{\prime \prime}(\tau)
\end{array}
$$

Here, $\hbar$ is Planck's constant divided by $2 \pi, k_{\mathrm{B}}$ is Boltzmann's constant, and $T$ is the temperature.

Following de Boeij et al., the coupling strength can be estimated from the width of the linear absorption spectrum, whereas the reorganization energy is linked to the Stokes shift between linear absorption and emission spectra. ${ }^{36,45}$ Analytical expressions for $M(t)$ can be derived within the multimode Brownian oscillator (MBO) model developed by Mukamel and co-workers. ${ }^{40}$ Finally, it should be mentioned that within the MBO treatment degrees of freedom that can couple to the 


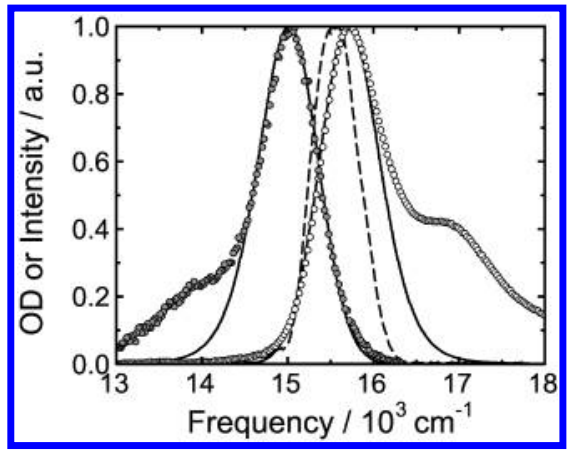

Figure 3. Linear absorption (open circles) and emission (gray circles) spectra of C1-DiD in bulk water. The power spectrum of the NOPA is shown as a dashed curve. The solid curves represent multimode Brownian oscillator simulations using a correlation function whose parameters are given in Table 1.

(artificial) electronic two-level system include not only those of the environment but also those of the chromophore itself. These give rise to a vibrational structure of the linear electronic spectra and to quantum beats in 4-wave mixing spectroscopies. ${ }^{40,47,48}$

\section{Results and Discussion}

1. C1-DiD in Bulk Water. The electronic absorption and emission spectra of C1-DiD in water at room temperature are displayed in Figure 3. The absorption spectrum peaks at 636 $\mathrm{nm}\left(15723 \mathrm{~cm}^{-1}\right)$ and has a full width at half-maximum (fwhm) of $40 \mathrm{~nm}\left(1000 \mathrm{~cm}^{-1}\right)$. Fitting only the low-energy edge with a Gaussian profile yields a fwhm of $790 \mathrm{~cm}^{-1}$. The emission spectrum is peaked at $665 \mathrm{~nm}\left(15038 \mathrm{~cm}^{-1}\right)$ with a fwhm of $800 \mathrm{~cm}^{-1}$ similar to the Gaussian width of the absorptive line shape. The Stokes shift amounts to $685 \mathrm{~cm}^{-1}$ giving a reorganization energy of $342.5 \mathrm{~cm}^{-1}$. For comparison, the NOPA power spectrum used for the 3PEPS measurements on C1-DiD in water is also shown in Figure 3. It is centered at $643 \mathrm{~nm}\left(15552 \mathrm{~cm}^{-1}\right)$ with a fwhm of $654 \mathrm{~cm}^{-1}$. This bandwidth fully captures the low-energy edge of the absorption spectrum. When the pure $0-0$ transition is located exactly between the spectral positions of the absorption and emission maxima, the laser detuning with respect to the electronic resonance amounts to $170 \mathrm{~cm}^{-1}$.

Representative normalized 3-pulse echoes on C1-DiD in bulk water are shown in Figure 4 for both conjugate phase matching directions of the triangular geometry. For comparison, an autocorrelation trace of the NOPA output is also shown. Each $t_{12}$ scan consisted of a total number of 1000 data points evenly distributed between -100 and +100 fs. The absolute time zero along $t_{12}$ was determined with an estimated accuracy of $\pm 1 \mathrm{fs}$ from the symmetry of the 2-pulse echoes, which were simultaneously recorded in the $2 k_{2}-k_{1}$ and $2 k_{1}-k_{2}$ directions. Furthermore, the time zero along $t_{23}$ was identified as the waiting period at which the 3-pulse echo peak shift became identical to the 2-pulse echo peak shift.

For a given $t_{23}$, the two conjugate 3 -pulse echoes possess mirror image symmetry because they only differ in the sign of the relative timing between the first and second pulses. To determine the echo peak shift for a given waiting delay, the peaks of conjugate echo pairs were fitted to a fourth-order polynomial as illustrated in Figure 5. For the fitting, only those data points were considered which exceeded a predefined threshold value of 0.8 of the normalized echo trace. Constraining the fit to the peak portion of the echo only is made possible by

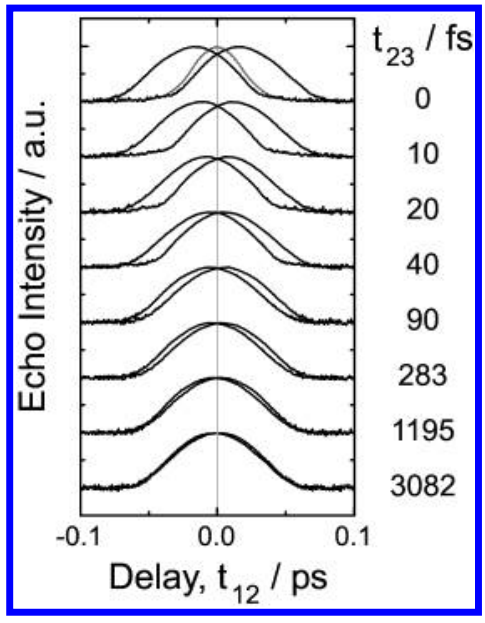

Figure 4. Series of 3-pulse photon echoes for C1-DiD in bulk water collected at representative waiting times, $t_{23}$. The echoes were detected in the $k_{4}$ and $k_{4}^{\prime}$ directions of the triangular phase-matching geometry (see Figure 1). An intensity autocorrelation of the NOPA pulses is shown as the gray curve.

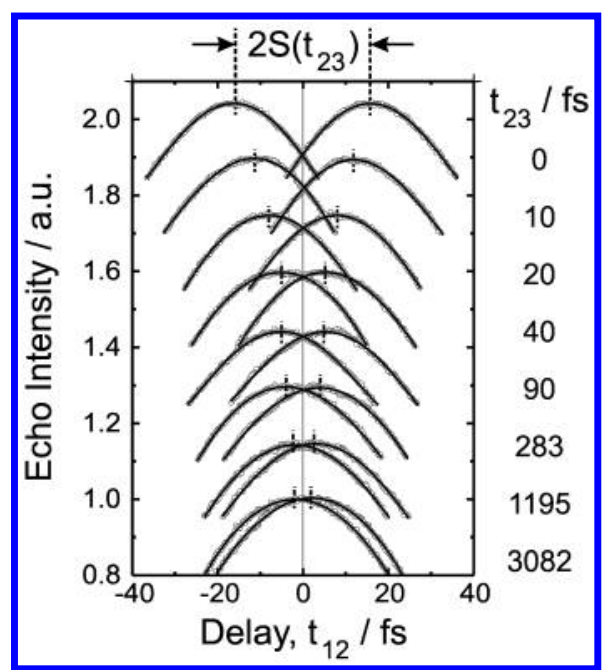

Figure 5. Polynomial fits (solid curves) to experimental 3-pulse photon-echoes (gray circles) for C1-DiD in bulk water collected at representative waiting times, $t_{23}$. The echoes were detected in the $k_{4}$ and $k_{4}^{\prime}$ directions of the triangular geometry. Note the decreasing echo peak shift with increasing $t_{23}$.

the large number of data points collected per $t_{12}$ scan. A threshold of 0.8 left more than 200 data points to be modeled by the polynomial function. This procedure was found superior to a simulation of the entire echo profile using either Gaussians or polynomials of various orders because of the pronounced asymmetry of the individual signals in particular at early waiting times (see Figure 4). The total echo peak shift, $S\left(t_{23}\right)$, was then measured as half the temporal delay between the maxima of the polynomial fits.

From both Figures 4 and 5, it is obvious that the echo peak shift decays as the waiting period is increased. Inspection of the raw data already indicates that ca. $50 \%$ of the initial 3PEPS disappears during the first $20 \mathrm{fs}$, whereas the remaining portion decays on time scales as long as several picoseconds. Furthermore, for waiting times longer than $3 \mathrm{ps}$, the peak shift has not yet fully vanished indicating that in liquid solution there is still some finite capability for rephasing and thus inhomogeneity left on such time scales. Figure 6 displays the 3PEPS as a function of the logarithmic waiting period. To quantify the time scales 


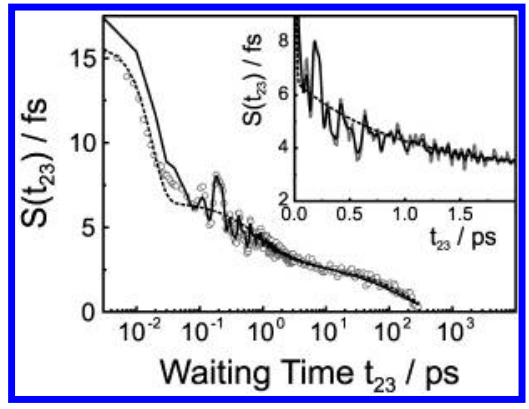

Figure 6. Experimental echo peak shift (gray circles) as a function of the waiting time for C1-DiD dissolved in bulk water. The dashed curve corresponds to the phenomenological fit of the data to a multiexponential decay (see eqn. 8). The solid curve represents a MBO simulation using the $M(t)$ from Table 1 . The inset emphasizes the short time evolution of the peak shift including the coherent wave packet contributions from the optical chromophore.

of the 3PEPS decay, the data are fitted to a sum of exponentials and a Gaussian (see Figure 6, dashed curve)

$$
S\left(t_{23}\right)=A_{\mathrm{G}} \exp \left(-t_{23}{ }^{2} / \tau_{\mathrm{G}}{ }^{2}\right)+\sum_{i=1}^{N} A_{\mathrm{I}} \exp \left(-t_{23} / \tau_{i}\right)
$$

To model the data satisfactorily three exponentials are required, i.e., $\mathrm{N}=3$. Their amplitudes, $A$, are $2.55,0.8$, and $3.2 \mathrm{fs}$ with corresponding decay times, $\tau$, of $180,6.0$, and $0.9 \mathrm{ps}$. The initial ultrafast decay is mimicked by the Gaussian whose amplitude, $A_{\mathrm{G}}$, is $9.25 \mathrm{fs}$ with a correlation time, $\tau_{\mathrm{G}}$, of $18 \mathrm{fs}$. The slow components with time constants in excess of 1 ps are needed to reproduce the tail seen for waiting times longer than roughly 2 ps. In particular, the intermediate exponential exhibits a time constant which is in good agreement with the time constant for single-molecule rotational diffusion of water as obtained by numerous other techniques for probing liquid dynamics. ${ }^{49-55}$ However, the contribution that is most typical for water is the dominant decay seen between $100 \mathrm{fs}$ and 2 ps. It is captured by the fastest exponential with a decay time of 0.9 ps. As we discussed previously, this component is reminiscent of the response by the intermolecular restricted translational modes of liquid water. ${ }^{33,51,52}$

Note that intramolecular vibrational coherences of the chromophore modulate the 3PEPS on time scales below 3 ps as illustrated in the inset of Figure 6 . The vibrational structure contributing to the electronic resonance of the chromophore was characterized in ref 33 by transient grating scattering (TG). The TG response contains seven underdamped intramolecular vibrational modes with frequencies below $600 \mathrm{~cm}^{-1}$. A Fourier analysis of the data displayed in Figure 6 demonstrates that the very same frequency components are also observed in the 3PEPS experiment as expected.

The nonoscillatory components from eq 8 and the oscillatory components of the TG data ${ }^{33}$ served as a first guess for the correlation function, $M(t)$. From this $M(t)$, a line broadening function was constructed according to eq 4 which was then used to compute the 3 PEPS profile..$^{40}$ Following ref 36 , the amplitudes of the individual Brownian oscillators were iteratively fine-tuned until reasonable agreement between the simulated and the experimental 3PEPS was accomplished. The coupling strength and the reorganization energy required for the $\mathrm{MBO}$ simulation were estimated from the linear spectra as described in ref 36. The simulation result is reproduced in Figure 6 as the solid curve. Except for waiting times shorter than $20 \mathrm{fs}$, the agreement is excellent. This also holds for a simulation of the linear
TABLE 1: Parameters of the Correlation Function Used for the Brownian Oscillator Simulations of the 3PEPS Data on C1-DiD Dissolved in Bulk Water at Room Temperature

\begin{tabular}{cccc}
\hline$M(t)$ & $\begin{array}{c}\text { relative coupling } \\
\text { strength }\end{array}$ & $\begin{array}{c}\text { frequency } \\
\omega / \mathrm{cm}^{-1}\end{array}$ & $\begin{array}{c}\text { damping or correl. } \\
\text { time, } \tau / \mathrm{ps}\end{array}$ \\
\hline underdamped $^{a}$ & 0.032 & 573 & 1.98 \\
& 0.012 & 549 & 3.63 \\
& 0.033 & 524 & 0.83 \\
& 0.052 & 313 & 0.65 \\
& 0.076 & 146 & 0.50 \\
overdamped $^{a}$ & 0.017 & 212 & 0.68 \\
& 0.102 & 32 & 0.31 \\
Gaussian $^{a}$ & 0.168 & & 1.0 \\
& 0.070 & & 7.5 \\
& 0.245 & & 180 \\
& 0.193 & & 0.014
\end{tabular}

${ }^{a}$ The functional forms of the correlation functions of the individual components to $M(t)$ can be found in ref 40 .

absorption and emission spectra (see Figure 3). Table 1 summarizes the resulting correlation function for C1-DiD in liquid water at room temperature. A detailed account including MBO simulations on the full temperature dependence of aqueous bulk solvation will be presented elsewhere.

Inspection of Table 1 illustrates that the correlation times of the overdamped modes agree very well with those from the multiexponential fit. This demonstrates that with the exception of the initial FID-type decay, the 3PEPS tracks the behavior of the correlation function, $M(t)$, quantitatively. The most prominent component to $M(t)$ is an overdamped mode with a correlation time of $1.0 \mathrm{ps}$. This mode gives rise to the pronounced decay of the 3PEPS between $100 \mathrm{fs}$ and $2 \mathrm{ps}$. Its time constant seems to be a universal time constant for liquid water as it was also found in numerous other studies on water dynamics. ${ }^{49-56}$ Similar time constants were also found in fluorescence upconversion experiments on aqueous bulk solvation of Coumarin dyes ${ }^{29,30}$ as well as in a 3PEPS study on the chromophore, eosin $\mathrm{Y}$, in bulk water. ${ }^{57}$ This 1 ps-overdamped oscillator reflects the collective response of the intermolecular restricted translational modes of the liquid, which are associated with bending and stretching motions of hydrogen bonds between nearest-neighbor molecules in the liquid. These modes are highly characteristic for the random network of $\mathrm{H}$ bonds that exists only in bulk water. We emphasize that such an assignment is in full agreement with computer simulations by Marcus and co-workers who concluded that subpicosecond solvation relaxation times are due to intermolecular nuclear degrees of freedom with higher frequencies. ${ }^{31,32}$ These can indeed only be those which are linked to hydrogen bond motions.

2. C18-DiD at DMPC-Water Interfaces. The linear spectra of C18-DiD in DMPC at room temperature are displayed in Figure 7. The absorption spectrum peaks at $648.6 \mathrm{~nm}$ (15 418 $\left.\mathrm{cm}^{-1}\right)$ and has a fwhm of $42.5 \mathrm{~nm}\left(1021 \mathrm{~cm}^{-1}\right)$. Fitting a Gaussian profile to the low-energy edge only yields a fwhm of $835 \mathrm{~cm}^{-1}$. The emission spectrum is peaked at $676.0 \mathrm{~nm}$ $\left(14793 \mathrm{~cm}^{-1}\right)$ with a fwhm of $865 \mathrm{~cm}^{-1}$, again very similar to the Gaussian width of the absorption spectrum. The Stokes shift amounts to $625 \mathrm{~cm}^{-1}$ giving a reorganization energy of $312.5 \mathrm{~cm}^{-1}$. To ensure similar excitation conditions as for C1-DiD in bulk water, the NOPA was tuned to a center wavelength of $656 \mathrm{~nm}$ corresponding to a detuning of $150 \mathrm{~cm}^{-1}$. Again, a NOPA bandwidth of $700 \mathrm{~cm}^{-1}$ (fwhm) allowed for a full spectral coverage of the low-energy edge of the absorption spectrum. 


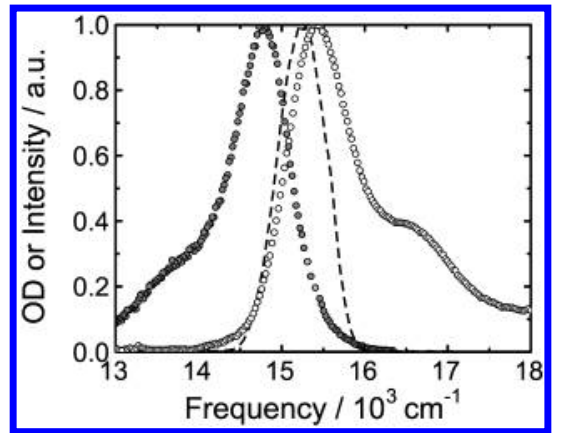

Figure 7. Linear absorption (open circles) and emission (gray circles) spectra of C18-DiD anchored to unilamellar vesicles of DMPC at 298 $\mathrm{K}$. The NOPA power spectrum is shown as a dashed curve.

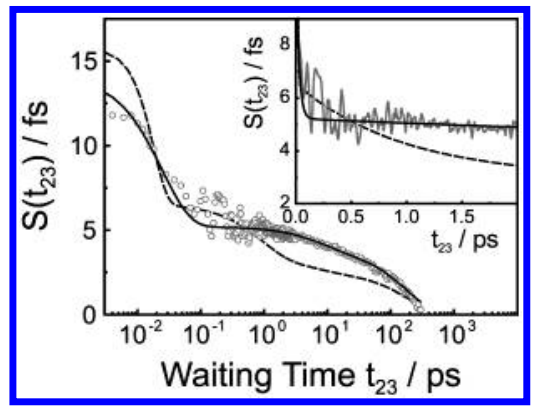

Figure 8. Echo peak shift (gray circles) as a function of the waiting time for C18-DiD anchored to unilamellar DMPC vesicles at room temperature. The solid line corresponds to a fit of the data to a multiexponential decay (see eq 8). For comparison, the corresponding fit to the bulk water data is also reproduced as the dashed curve. The inset emphasizes the chromophore wave packet contributions.

Both the bandwidths and the Stokes shift at the lipid interface are very similar to those obtained on the water-soluble but otherwise identical optical chromophore in the bulk. Only a slight red-shift of about $274 \mathrm{~cm}^{-1}$ distinguishes the electronic interfacial resonance from that in the bulk which indicates a higher polarity to which the chromophore is exposed at the interface.

Figure 8 shows the results of the 3PEPS experiment performed on C18-DiD anchored to DMPC-water interfaces at room temperature. Again, a multiexponential decay according to eq 8 is fitted to the membrane data. Contrary to bulk water, only two exponentials are needed to satisfactorily reproduce the data, i.e., $N=2$. Their time constants are 180 and 8 ps with amplitudes of 3.9 and $1.3 \mathrm{fs}$, respectively. The early time portion below $100 \mathrm{fs}$ is well captured by a Gaussian component whose correlation time is 25 fs with an amplitude of 9 fs. For comparison, the multiexponential fit to the bulk water data is also reproduced as the dashed curve. The somewhat smaller magnitude of the peak shift at early times compared to water can be attributed to the slightly different coupling strengths in combination with detuning effects. Overall, the decay of the echo peak shift at the membrane interface is dramatically decelerated in comparison to the aqueous bulk phase. Only the exponential component with a time constant of 8 ps seems reminiscent for some diffusive bulklike contributions, which might by traced back to water rotational motion. In fact, the correlation time agrees almost quantitatively with the Debye relaxation time of water obtained from most recent complex dielectric permittivity spectra of unilamellar suspensions of DMPC.$^{58}$ In an earlier publication, we tentatively assigned this component to diffusive reorientational motion of interstitial water molecules, which can penetrate into the membrane headgroup region. ${ }^{33}$ There is, however, evidence from molecular

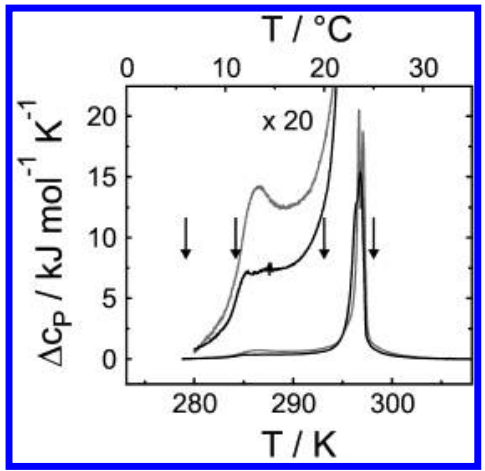

Figure 9. Differential specific heat of labeled (black) and unlabeled (gray) unilamellar DMPC vesicles in aqueous solutions. The magnified portion of the data emphasizes the pretransition separating the $L_{\beta^{\prime}}$ and $P_{\beta^{\prime}}$ phases. Vertical arrows indicate temperatures at which 3PEPS experiments were performed.

dynamics (MD) simulations ${ }^{23,24,26,27}$ that this motion is considerably slower at the membrane interface in comparison to the bulk (see below).

More importantly, however, Figure 8 demonstrates that the pronounced decay of the water data in the temporal window between $100 \mathrm{fs}$ and $2 \mathrm{ps}$ is entirely missing at the lipid interface. Instead, the interfacial 3PEPS is rather constant in this temporal region and begins to decay appreciably only for waiting times longer than 2 ps. As discussed in the previous section, the echo peak shift decay on time scales below 2 ps is highly characteristic for the random hydrogen-bonded network of the bulk liquid. The obvious discrepancy between the 3PEPS data obtained at the interface and those in the bulk phase on exactly these time scales gives some evidence for a substantial disruption of the hydrogen-bond network of water near the interface to the phospholipid membrane. At any rate, the above result proves that, at room temperature, membrane bound C18-DiD is entirely insensitive to any solvation dynamics originating from the restricted translational modes typical for the bulk phase of water.

3. Temperature-Dependent 3PEPS at DMPC-Water Interfaces. To characterize the thermodynamic phase behavior of the labeled and unlabeled vesicles, differential scanning calorimetry was carried out. The temperature-dependent differential specific heat for DMPC in the absence and in the presence of the optical chromophore is shown in Figure 9. The most remarkable feature for the unlabeled vesicles are the two pronounced peaks around $23.7^{\circ} \mathrm{C}$ which indicate the so-called chain-melting transition. ${ }^{3,35,59}$ The splitting of this phase transition into two separate peaks is linked to the extrusion techniques by which the vesicles were prepared and is probably connected to liposomal structural transitions. ${ }^{35}$ The highly cooperative chain melting region separates the liquid-crystalline (or fluid) phase denoted $L_{\alpha}$ from the gel phases of the lipid. Above the melting region, the alkyl tails in the core of the membrane are fully disordered similar to a liquidlike state. ${ }^{19,20,35}$

Two different gel phases can be distinguished whose coexistence region is indicated by a broad local maximum of the specific heat around $13{ }^{\circ} \mathrm{C}$. The low-temperature phase is called the gel phase, $L_{\beta^{\prime}}$, and displays narrow wide-angle X-ray scattering peaks due to long-range order within the membrane plane consistent with a parallel packing of the alkyl tails in a nearly two-dimensional hexagonal lattice. Furthermore, the hydrocarbon tails are tilted by a few degrees with respect to the membrane normal. However, diffuse X-ray background scattering on the $L_{\beta^{\prime}}$ phase indicates the presence of residual structural disorder originating from the headgroup region of the membrane. ${ }^{19,20,35}$ 
The high-temperature phase is termed $P_{\beta^{\prime}}$ and displays defect regions in the hydrocarbon core in which the alkyl tails are partially melted (e.g., disordered) similar to the fluid phase. The defect regions are accompanied by the formation of periodic rippled structures ${ }^{19,20,35}$ on the membrane surface with ripple periods of a few hundred $\AA$ as seen in freeze-fracture electron microscopy. Recent suggestions by Heimburg provide a direct link between ripple formation and the chain melting process. ${ }^{60}$ Monte Carlo simulations employing geometrical constraints due to the closed vesicular topology of the membrane show indeed the formation of a periodic pattern of fluid and gel-rich domains. Because the occupied area per lipid changes upon melting by about $24 \%$, the formation of a fluid line defect is energetically favorable resulting in a local bending of the membrane equivalent to a ripple. ${ }^{60}$ The $P_{\beta^{\prime}} \leftrightarrow L_{\beta^{\prime}}$ interconversion observed around $13{ }^{\circ} \mathrm{C}$ is also called the rippled gel-to-gel transition or pre-transition. ${ }^{35}$

Only small changes of the $\Delta c_{\mathrm{P}}$ traces are observed upon labeling the DMPC vesicles (see Figure 9). Most remarkably, the splitting of the melting region into two separate peaks is less pronounced resulting in a single maximum at $23.7^{\circ} \mathrm{C}$. However, a distinct shoulder at $23.4{ }^{\circ} \mathrm{C}$ can still be resolved. Hence, the melting region has moved to lower temperatures by roughly $0.3{ }^{\circ} \mathrm{C}$. Furthermore, the pretransition is also less pronounced and seems to be shifted by $1-2{ }^{\circ} \mathrm{C}$ to lower temperatures as judged by the inflection point of the $\Delta c_{\mathrm{P}}$ trace of the labeled vesicles in this temperature range. The depression of the chain melting temperature together with the broadening of the transition region is in accordance with previous DSC data on DPPC bilayers ${ }^{61}$ labeled with indocarbocyanine dyes $\mathrm{Cn}$ DiD of different alkyl chain lengths, $n=12-22$. In combination with fluorescence quenching experiments, these DSC data were interpreted with a slight preference of the optical chromophore to partition into the gel phase at room temperature. ${ }^{62}$ The same conclusion was drawn from fluorescence quenching experiments on C18-DiD in DMPC membranes at different chromophore concentrations. ${ }^{63}$ In fact, this partitioning behavior was exploited in recent attempts at confocal imaging of phase separation and domain formation in giant unilamellar vesicles of binary lipid mixtures using fluorescence from the chromophore, C20-DiD. ${ }^{34}$

To explore manifestations of this lipid polymorphism in the short-time dynamics of solvation of C18-DiD at aqueous interfaces of DMPC, photon-echo experiments were performed in each of the three phases of the vesicles. Prior to the nonlinear studies, however, the temperature dependence of the linear resonance was investigated in more detail. In turns out that the spectral position of the absorption spectrum is highly sensitive to the temperature as depicted in Figure 10.

In the fluid phase, the peak position of the absorption spectrum is fairly constant. The resonance suddenly shifts by more than $40 \mathrm{~cm}^{-1}$ to lower frequencies at the chain melting temperature. This red-shift is a direct consequence of the reduction of the occupied area per lipid in going from the fluid to the gel phase. The enhanced packing efficiency of the lipids in the gel phases results in a higher local polarity experienced by the optical chromophore as compared to the liquid-crystalline phase. In the gel phases, only a continuous red shift of the resonance with decreasing temperature is observed according to a temperature coefficient of about $-1.21 \mathrm{~cm}^{-1} / \mathrm{K}$. Interestingly, the pretransition does not lead to deviations from this linear temperature dependence. Hence, the polarity smoothly varies through the pretransition without discontinuity. Because the total spectral shift in the gel phases only amounts to 20 $\mathrm{cm}^{-1}$, detuning effects on the 3PEPS experiment can be

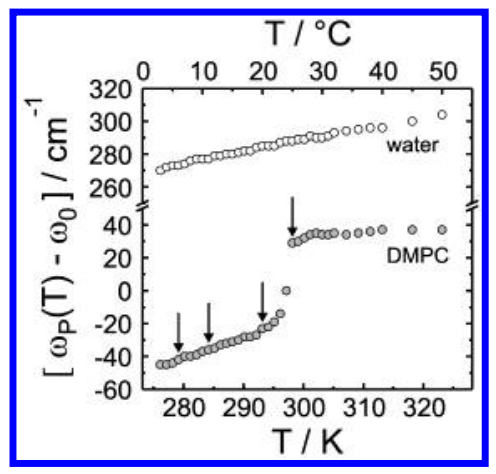

Figure 10. Spectral position of the absorption spectrum of membrane bound C18-DiD and aqueous C1-DiD. The data are referenced to $\omega_{0}$ $=15418 \mathrm{~cm}^{-1}$ corresponding to the spectral position of C18-DiD in DMPC at $298 \mathrm{~K}$. The arrows indicate temperatures at which 3PEPS experiments were performed.

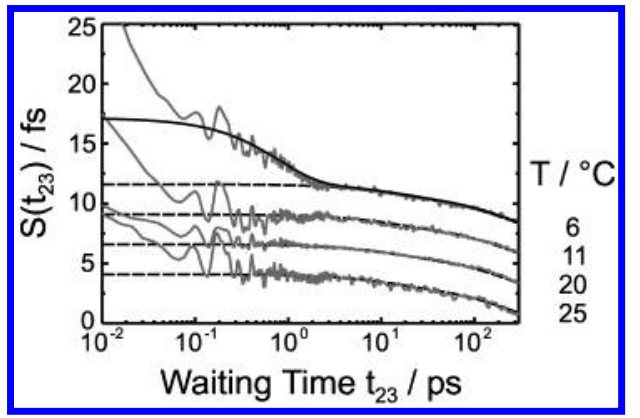

Figure 11. Temperature-dependent 3PEPS (gray curves) as a function of the waiting time for C18-DiD at aqueous interfaces of unilamellar DMPC vesicles. Phenomenological fits of the data to a biexponential (dashed) and a triple-exponential (solid black) decay are also shown.

neglected. For comparison, the temperature-dependent spectral position of C1-DiD in bulk water is also reproduced in Figure 10. Over the entire temperature range, its electronic resonance is blue-shifted by more than $250 \mathrm{~cm}^{-1}$ with respect to membrane bound C18-DiD. Here, the center frequency decreases linearly upon cooling according to a temperature coefficient of only $-0.70 \mathrm{~cm}^{-1} / \mathrm{K}$. Both findings imply that, regardless of the thermodynamic phase of the lipid bilayer, the chromophore never leaves the interface to fully enter the aqueous bulk phase.

The temperatures at which 3PEPS measurements were carried out are indicated in Figures 9 and 10 by arrows. The corresponding data are summarized in Figure 11 as gray curves. Note that for clarity, the 3PEPS traces were tail-matched and vertically shifted by an increment of $2.5 \mathrm{fs}$. Because our focus is on solvent dynamics only, the initial FID-type decay of the 3PEPS will be disregarded in the following. Again, a phenomenological fit to a biexponential decay is displayed as dashed curves. Temperature independent time constants of 180 and 8 ps with corresponding amplitudes of 3.9 and 1.3 fs were used to simultaneously fit all four 3PEPS traces. There are two issues which are most remarkable:

(i) Because within the experimental accuracy the 3PEPS profiles obtained at 25,20 , and $11{ }^{\circ} \mathrm{C}$ are identical, the $L_{\alpha} \leftrightarrow$ $P_{\beta^{\prime}}$ transition (i.e., fluid-to-rippled gel) does not affect the solvation dynamics at all. At first, this seems to contradict the behavior of the spectral position of the absorption spectrum which changes most dramatically upon chain melting. However, linear spectra do not contain any information regarding line broadening dynamics. The spectral position is merely sensitive to the time-averaged polarity at the location of the chromophore as defined by the lipid packing efficiencies of the fluid and the 


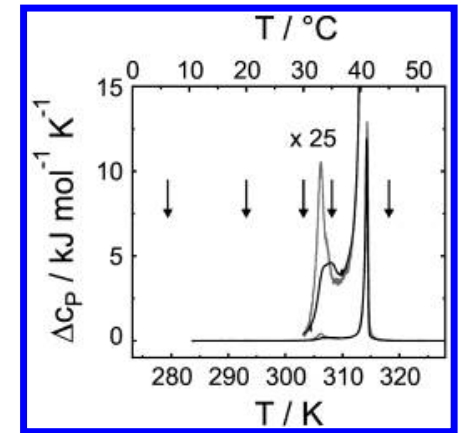

Figure 12. Differential specific heat of labeled (black) and unlabeled (gray) unilamellar DPPC vesicles in aqueous solutions. Vertical arrows indicate the temperatures at which 3PEPS data were collected.

gel-phases. Chain melting modifies primarily the dynamics of the membrane interior; that is, the collection of intra- and intermolecular modes of the hydrophobic core resembles either those of a liquid or those of a solid depending upon the packing mode of the alkyl tails. However, Figure 11 shows quite convincingly that structural dynamics in the rippled-gel and the fluid phase are indistinguishable by the 3PEPS even on time scales as long as $0.3 \mathrm{~ns}$. Therefore, the insensitivity of the 3PEPS to the main phase transition gives direct evidence for the chromophore being indeed exclusively located at the interface.

(ii) As opposed to the main phase transition, the rippled gelto-gel pretransition strongly affects the 3PEPS decay. In particular, the 3PEPS profile obtained in the gel phase deviates from the biexponential fit on time scales below $2 \mathrm{ps}$. To adequately reproduce the $6{ }^{\circ} \mathrm{C}$ data, a third exponential is needed whose time constant is $0.8 \mathrm{ps}$ (amplitude of $5.5 \mathrm{fs}$ ) very similar to the fastest exponential observed for C1-DiD in bulk water (see Figure 6). Therefore, one might conclude that, at sufficiently low temperatures, the chromophore leaves the interface and enters the aqueous bulk phase far above the membrane surface. However, because the spectral position of the linear absorption of $\mathrm{C} 18$-DiD does not change at all during the pretransition and remains red-shifted to that of C1-DiD in bulk water (see Figure 10), this scenario seems unlikely. Therefore, in contrast to the main phase transition, the pretransition must affect ordering phenomena and structural relaxations connected with the interface region of the membrane and modifies the solvation response of the chromophore located exclusively at that interface.

4. Temperature-Dependent 3PEPS at DPPC-Water Interfaces. To confirm whether this peculiar temperature dependence of the 3PEPS is indeed directly linked to the polymorphism of the lipid membrane, complementary echo experiments were performed on unilamellar vesicles of DPPC labeled with the same optical chromophore. Again, prior to the nonlinearoptical experiments, DSC experiments were carried out to characterize the thermodynamic phase behavior of both the labeled and unlabeled vesicles (see Figure 12).

It can be seen that the entire phase diagram of DPPC is shifted to significantly higher temperatures with respect to that of DMPC. The main chain-melting transition is now located at $41.2{ }^{\circ} \mathrm{C}$ for the unlabeled and $41.1{ }^{\circ} \mathrm{C}$ for the labeled vesicles. Furthermore, the pretransition is found around $33.0^{\circ} \mathrm{C}$ resulting in a slightly narrower stability range of the rippled-gel phase compared to the shorter chain lipid. Hence, the $L_{\alpha} \leftrightarrow P_{\beta^{\prime}}$ transition is shifted by roughly 17 degrees and the $P_{\beta^{\prime}} \leftrightarrow L_{\beta^{\prime}}$ transition by approximately 20 degrees to higher temperatures as compared to DMPC.

Again, photon echo measurements were carried out in each of the three phases as indicated by the vertical arrows in Figure

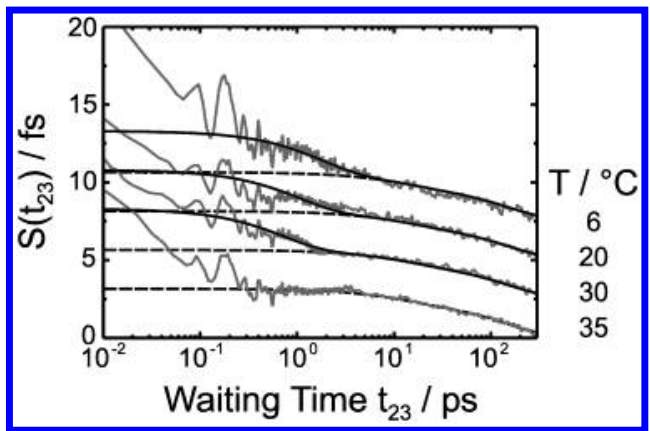

Figure 13. Temperature-dependent 3PEPS (gray curves) as a function of the waiting time for C18-DiD anchored to aqueous interfaces of unilamellar DPPC vesicles. Also shown are phenomenological fits of the data to a biexponential (dashed) and a triple-exponential (solid black) decay.

12. The corresponding 3PEPS traces are displayed in Figure 13. For clarity, the data were again tail-matched and incrementally shifted by $2.5 \mathrm{fs}$ along the $S\left(t_{23}\right)$ axis. The fluid phase data taken at $45{ }^{\circ} \mathrm{C}$ were omitted for clarity but are within the signalto-noise ratio identical to those obtained in the rippled-gel phase at $35^{\circ} \mathrm{C}$. The latter can be fitted reasonably well over a waiting period from 100 fs to 300 ps using a biexponential decay whose time constants are 160 and 12 ps with corresponding amplitudes of 2.3 and $0.85 \mathrm{fs}$. The very same biexponential decay is also able to reproduce the long-time tail (i.e., $t_{23}>3 \mathrm{ps}$ ) of the 3PEPS profiles obtained at the lower temperatures.

Although not as pronounced as in DMPC, one can again clearly see that the experimental data obtained below the pretransition deviate significantly from this biexponential fit on time scales shorter than 2 ps. Again a third exponential component is necessary to satisfactorily reproduce the data. Its time constant varies from $0.7 \mathrm{ps}$ at $25^{\circ} \mathrm{C}$ and $1.0 \mathrm{ps}$ at $20^{\circ} \mathrm{C}$ to $1.7 \mathrm{ps}$ at $6{ }^{\circ} \mathrm{C}$, whereas its amplitude is $2.65 \mathrm{fs}$ independent of the temperature. The time constants are again very similar to those obtained for C1-DiD in bulk water. It should be noted, however, that this ultrafast component significantly deviates from an exponential and that a Gaussian functional form might be used for fitting equally well.

Most importantly and fully in line with the DMPC data, this third component is detected neither in the fluid nor in the rippled-gel phase and is only visible in the gel phase, $L_{\beta^{\prime}}$. This finding strongly supports the notion that this particular response on time scales below 2 ps is indeed entirely gel-phase specific. Therefore, one can confidently conclude that the rippled gelto-gel phase transition affects ordering phenomena in the interface region which in turn are associated with pronounced modifications of structural fluctuations occurring specifically at the interface of the membrane bilayer.

5. Origin of the Gel-Phase Specific Solvation Response. Having established its gel-phase specific nature, the microscopic origin of the ultrafast sub-2-ps response still needs to be clarified. Although according to most recent X-ray scattering experiments ${ }^{64}$ and theoretical models, ${ }^{60}$ the transition from $L_{\beta^{\prime}}$ to $P_{\beta^{\prime}}$ is likely to be directly coupled to partial chain melting and formation of fluid-rich rippled defects, there are several indications that the same transition is also linked to ordering phenomena in the headgroup region. ${ }^{19,20,35}$

First, the formation of a rippled phase and hence the existence of a pretransition depends on the level of hydration. It cannot be observed in fully dehydrated membranes. Infrared spectroscopy on multilamellar DPPC vesicles ${ }^{65}$ demonstrates that upon cooling the $P_{\beta^{\prime}}$ phase, vibrational transitions of the lipids decrease at the expense of band intensities associated with the 
intramolecular $\mathrm{OH}$ stretch of water molecules. This finding was interpreted with an increase of the thickness of the interlamellar water layer because of headgroup dehydration on aproaching the gel phase. More specifically, by using a dual radiolabel centrifugal technique, Channareddy and Janes ${ }^{66}$ deduced a number of 8.2 tightly bound water molecules per lipid for multilamellar DPPC vesicles in the rippled-gel phase, whereas in the gel phase, this number is reduced to 4.7. Hence in this respect, the rippled-gel phase is similar to the liquid-crystalline phase, $L_{\alpha}$, for which 8.6 strongly interacting water molecules per lipid were found. Of course, such a division of water molecules into "free" (i.e., bulk) and "bound" (i.e., interfacial) has to take into account the time scale on which a chemical exchange between these two species can occur. ${ }^{67}$ Nonetheless, it is commonly accepted that the rippled-gel phase is hydrated similar to the fluid phase but significantly better hydrated than the pure gel phase. The preferential binding sites for water are the ester carbonyls, phosphate, and $N$-methyls; that is, water distributes exclusively in the headgroup region. ${ }^{68}$

NMR has been widely used to extract dynamical information on the polar headgroups of lipids ${ }^{69}$ In particular, deuterium quadrupole splittings and spin-lattice relaxation rates for selectively labeled headgroup segments have been studied in multilamellar liposomes as a function of water content. ${ }^{70,71}$ The $\alpha$ and $\beta$ segments of the choline moiety were characterized by effective correlation times for isotropic tumbling motion of about $100 \mathrm{ps}$ at full hydration, whereas the correlation times of $\gamma$ segments were roughly a factor of 2 faster because of the internal motion of the terminal $N$-methyl rotor. The dependence of all three correlation times on the water content was almost identical implying that the backbone of the choline headgroup moves more or less as one unit. ${ }^{71}$ Increasing the level of hydration resulted in a dramatic increase of the rate of headgroup motion which was explained by steric arguments: water uptake by the polar region of the bilayer surface causes a progressive loosening up of the lipid headgroup packing and an increased motional freedom. ${ }^{71}$ Notice that the 3PEPS data shown in Figures 11 and 13 exhibit quite the opposite behavior. The 3PEPS decay for $0.05 \mathrm{ps} \leq t_{23} \leq 2 \mathrm{ps}$ is dramatically accelerated when going from the rippled-gel phase to gel phase. In other words, the nuclear degrees of freedom responsible for the fast exponential component (correlation time of about $1 \mathrm{ps}$ ) are essentially frozen in the better hydrated rippled-gel phase and fluid phase. Such a finding clearly rules out an assignment of this component to motions of the lipid headgroups.

The translational and reorientational dynamics of water molecules at aqueous lipid-membrane interfaces has also been studied by NMR spectroscopy. Pulsed field gradient proton NMR was used by Wassall ${ }^{72}$ as well as by Volke et al. ${ }^{73}$ to determine translational diffusion coefficients of water in multilamellar palmitoyl-oleoyl (POPC), dioleoyl (DOPC), egg yolk phosphatidylcholine (lecithin), and DPPC dispersions. In parallel, deuterium quadrupole splittings and spin lattice relaxation rates have been measured to obtain information on the rotational motion of water. The results were interpreted with a model that assumes a perturbation of the water dynamics by the lipid interface. ${ }^{73}$ The perturbation decays exponentially with increasing hydration and interpolates between the rotational (translational) correlation time, $\tau_{\mathrm{S}}$, of water molecules directly bound to the lipid headgroups and the rotational (translational) correlation time, $\tau_{\mathrm{f}}$, of free (i.e., interlamellar) water molecules. The quantity, $\tau_{\mathrm{S}}$, was found to be of the order of $100 \mathrm{ps}$, whereas $\tau_{\mathrm{f}}$ varied between 5 and $10 \mathrm{ps}$ similar to the value found for the bulk liquid. From these values a lateral diffusion coefficient of
$0.1-0.2 \times 10^{-5} \mathrm{~cm}^{2} / \mathrm{s}$ was deduced at low levels of hydration (i.e., 5 water molecules per lipid) $)^{72}$ which is equivalent to a period of roughly $1 \mathrm{~ns}$ required for a water molecule to explore the area of the lipid headgroup. In summary, the presence of the lipid surface decelerates the translational motion of water by one order and the rotational motion by 2 orders of magnitude compared to the bulk. Interestingly, $\tau_{\mathrm{S}}$, was very similar to the correlation time for tumbling motion of the choline unit indicating that water reorientation is highly correlated with headgroup motion. ${ }^{73}$ Similar to the headgroups motions (see above), an increase of the water content increases the rate for water reorientations and translational jumps. ${ }^{72,73}$ Once again, this finding is in contrast to our 3PEPS data implying that water molecules which are tightly bound to the headgroups cannot contribute to the gel-phase specific sub-2-ps solvation response of C18-DiD.

Because the 3PEPS of C18-DiD is apparently not sensitive (i) to motions in the hydrocarbon core, (ii) to motions related to the headgroup, and (iii) to motions of water molecules which are tightly bound to the headgroups, the gel-phase specific sub2-ps component can only originate from weakly interacting water molecules. However, because the polarity sensed by $\mathrm{C} 18$ DiD is governed by lipid packing and because this polarity differs significantly from the aqueous bulk phase (as evidenced by the temperature-dependent spectral position of the linear absorption), these weakly interacting water molecules have to be distinguished from those of the bulk. Evidently, the formal division into two distinct water species only (i.e., "bound" and "free") is too simplistic.

All our findings taken together strongly suggest that the chromophore becomes sensitive to an intermediate water layer when the membrane is in the pure gel phase, $L_{\beta^{\prime}}$. This intermediate water layer is located between the layer of tightly bound headgroup water and the true aqueous bulk phase far away from the corrugated membrane surface. The layer may only be formed when the membrane assumes its $L_{\beta^{\prime}}$ phase with the lipid headgroups being dehydrated (i.e., $4-5$ water molecules per lipid as opposed to 8-9 for $P_{\beta^{\prime}}$ and $L_{\alpha}$ ). Alternatively, the intermediate layer may exist regardless of the thermodynamic phase of the lipid but the chromophore's distribution along the bilayer normal is shifted to larger distances upon headgroup dehydration (i.e., the chromophore is slightly expulsed from the interface). Despite a hydrogen-bonded network which is perturbed by the lipid headgroups, water molecules of the intermediate layer exhibit a motional freedom similar to the bulk. This might explain a strongly red-shifted resonance in comparison to the aqueous bulk phase as well as the occurrence of a gel-phase specific contribution to the 3PEPS decay whose time constant is reminiscent of bulk water.

Obviously, the 3PEPS experiment does not contain information regarding the chromophore's distribution along the bilayer normal. However, MD simulations clearly capture the different degrees of headgroup hydration depending upon the thermodynamic phase of the bilayer. ${ }^{21,22}$ From such calculations, it was found that the transition from interfacial to bulk water occurs on much larger length scales for the fluid phase as compared to the gel phase making the formation of an intermediate water layer actually more reasonable for the high-temperature phases. Unfortunately, we are not aware of equivalent MD simulations on the rippled-gel phase of phospholipid membranes. To our knowledge, dynamical information with sufficient spatial resolution such as reorientational or translational correlation times as a function of the distance from the membrane surface have not been retrieved from MD trajectories so far. However, we hope 
to perform equivalent 3PEPS experiments soon on suitably synthesized optical chromophores whose distances can be varied systematically along the membrane normal. This may be very helpful in quantifying the spatial dimensions over which the transition from tightly bound interfacial solvent water to bulk solvent water above phospholipid membrane surfaces occurs.

\section{Conclusions}

In summary, we have performed the temperature-dependent photon-echo studies on a cyanine dye which is noncovalently anchored to aqueous lipid bilayer interfaces. These systematic studies were aimed at uncovering manifestations of the thermodynamic phase behavior of synthetic lipid membranes in ultrafast structural relaxations relevant to solvation occurring specifically at their interfaces to water.

In particular, it was found that the 3PEPS is insensitive to the main phase transition separating the gel and the fluid phases of the membrane regardless of the chemical composition of the lipid. This insensitivity is expected because (i) the chromophore resides preferentially at the aqueous interface of the membrane and (ii) the main phase transition is primarily connected with ordering phenomena in the hydrophobic core of the lipid bilayer. In contrast to the main phase transition, the 3PEPS is highly sensitive to the pretransition separating the rippled-gel phase from the pure gel-phase of the membrane. This sensitivity reflects pretransition-induced ordering phenomena occurring specifically at the interface of the membrane to the surrounding water. A highly characteristic component to the 3PEPS decay on a time scale below 2 ps can only be found when the membrane is in the gel phase. This ultrafast contribution can neither be observed in the rippled-gel nor in the fluid phase. By comparison with complementary 3PEPS experiments on the same optical chromophore dissolved in bulk water, it was concluded that this component reflects dynamics of weakly bound water in the vicinity of the membrane which is to be distinguished from the aqueous bulk phase.

In combination with MD simulations, the 3PEPS method may be helpful in unraveling the transverse organization of water above lipid membranes. In the future, this technique may be able to monitor water dynamics near biological boundaries with high spatial resolution provided suitable optical chromophores are designed whose distance along the membrane normal can be well-controlled. In light of the results presented here, a division of water into "free" and "tightly bound" species only seems insufficient.

Acknowledgment. Financial support by the Deutsche Forschungsgemeinschaft through the SFB 357 "Molecular Mechanisms of Unimolecular Processes" is gratefully acknowledged.

\section{References and Notes}

(1) Membrane Permeability: 100 Years since Ernest Overton; Deamer, D. W., Kleinzeller, A., Fambrough, D. M., Eds.; Academic Press: San Diego, CA, 1999; Vol. 48.

(2) Membrane Spectroscopy; Grell, E., Ed.; Springer-Verlag: Berlin, 1981; Vol. 31.

(3) Cevc, G.; Marsh, D. Phospholipid bilayers: Physical principles and models; John Wiley \& Sons: New York, 1987.

(4) Nandi, N.; Bhattacharyya, K.; Bagchi, B. Chem. Rev. 2000, 100, 2013.

(5) Castner, E. W., Jr.; Maroncelli, M. J. Mol. Liq. 1998, 77, 1.

(6) Castner, E. W., Jr.; Bagchi, B.; Maroncelli, M.; Webb, S. P.; Ruggiero, A. J.; Fleming, G. R. Ber. Bunsen-Ges. Phys. Chem. 1988, 92, 363.

(7) Jarzeba, W.; Walker, G. C.; Johnson, A. E.; Barbara, P. F. Chem. Phvs. 1991, 152, 57.
(8) Kahlow, M. A.; Jarzeba, W.; Kang, T. J.; Barbara, P. F. J. Chem. Phvs. 1989, 90, 151.

(9) Kovalenko, S. A.; Ruthmann, J.; Ernsting, N. P. Chem. Phys. Lett. 1997, 271, 40.

(10) Sykora, J.; Kapusta, P.; Fidler, V.; Hof, M. Langmuir 2002, 18, 571

(11) Hutterer, R.; Parusel, A. B. J.; Hof, M. J. Fluoresc. 1998, 8, 389

(12) Hutterer, R.; Schneider, F. W.; Sprinz, H.; Hof, M. Biophvs. Chem. 1996, 61, 151 .

(13) Krasnowska, E. K.; Gratton, E.; Parasassi, T. Biophys. J. 1998 , $74,1984$.

(14) Levinger, N. E. Curr. Opin. Coll. Interface Sci. 2000, 5, 118.

(15) Pal, S. K.; Sukul, D.; Mandal, D.; Sen, S.; Bhattacharyya, K. Chem. Phys. Lett. 2000, 327, 91

(16) Pal, S. K.; Sukul, D.; Mandal, D.; Sen, S.; Bhattacharyya, K. Tetrahedron 2000, 56, 6999.

(17) Datta, A.; Pal, S. K.; Mandal, D.; Bhattacharya, K. J. Phys. Chem. B 1998, 102, 6114.

(18) Pal, S. K.; Sukul, D.; Mandal, D.; Bhattacharyya, K. J. Phys. Chem. B 2000, 104, 4529 .

(19) Nagle, J. F.; Tristram-Nagle, S. Curr. Opin. Struct. Biol. 2000, 10, 474.

(20) Nagle, J. F.; Tristram-Nagle, S. Biochim. Biophys. Acta 2000, 1469, 159.

(21) Tu, K.; Tobias, D. J.; Klein, M. L. Biophys. J. 1995, 69, 2558.

(22) Tu, K. C.; Tobias, D. J.; Klein, M. L. J. Phys. Chem. 1995, 99, 10035 .

(23) Heller, H.; Schaefer, M.; Schulten, K. J. Phys. Chem. 1993, 97, 8343 .

(24) Zhou, F.; Schulten, K. J. Phys. Chem. 1995, 99, 2194.

(25) Tieleman, D. P.; Berendsen, H. J. C. J. Chem. Phys. 1996, 105, 4871.

(26) Alper, H. E.; Bassolino-Klimas, D.; Stouch, T. R. J. Chem. Phys 1993, 99, 5547.

(27) Alper, H. E.; Bassolino-Klimas, D.; Stouch, T. R. J. Chem. Phys 1993, 98, 9798.

(28) Epand, R. M.; Kraayenhof, R. Chem. Phys. Lipids 1999, 101, 57. (29) Nagarajan, V.; Brearley, A. M.; Kang, T.-J.; Barbara, P. F. J. Chem. Phys. 1987, 86, 3183.

(30) Jimenez, R.; Fleming, G. R.; Kumar, P. V.; Maroncelli, M. Nature 1994, 369, 471

(31) Song, X. Y.; Chandler, D.; Marcus, R. A. J. Phys. Chem. 1996, 100,11954

(32) Hsu, C. P.; Song, X. Y.; Marcus, R. A. J. Phys. Chem. B 1997, 101, 2546.

(33) Bürsing, H.; Ouw, D.; Kundu, S.; Vöhringer, P. Phys. Chem. Chem. Phvs. 2001, 3, 2378.

(34) Schwille, P.; Korlach, J.; Webb, W. W. Cytometry 1999, 36, 176.

(35) Koynova, R.; Caffrey, M. Biochim. Biophvs. Acta 1998, 1376, 91.

(36) de Boeij, W. P.; Pshenichnikov, M. S.; Wiersma, D. A. J. Phys. Chem. 1996, 100, 11806.

(37) Passino, S. A.; Nagasawa, Y.; Joo, T.; Fleming, G. R. J. Phys. Chem. 1997, 101, 725 .

(38) Bürsing, H. Dissertation, University of Göttingen, Germany, 2002. (39) MacDonald, R. C.; MacDonald, R. I.; Menco, B. P. M.; Takeshita, K.; Subbarao, N. K.; Hu, L. Biochim. Biophys. Acta 1991, 1061, 297.

(40) Mukamel, S. Principles of nonlinear optical spectroscopy; Oxford University Press: New York, 1995.

(41) de Boeij, W. P.; Pshenichnikov, M. S.; Wiersma, D. A. Annu. Rev. Phys. Chem. 1998, 49, 99.

(42) Fleming, G. R.; Joo, T.; Cho, M. Adv. Chem. Phys. 1997, 101, 141 (43) Fleming, G. R.; Passino, S. A.; Nagasawa, Y. Philos. Trans. R. Soc. London A 1998, 356, 389.

(44) Fleming, G. R.; Cho, M. Annu. Rev. Phys. Chem. 1996, 47, 109 (45) de Boeij, W. P.; Pshenichnikov, M. S.; Wiersma, D. A. Chem. Phys. Lett. 1996, 253, 53.

(46) Book, L. D.; Scherer, N. F. J. Chem. Phys. 1999, 111, 792.

(47) Ohta, K.; Larsen, D. S.; Yang, M.; Fleming, G. R. J. Chem. Phys. 2001, 114, 8020 .

(48) Larsen, D. S.; Ohta, K.; Xu, Q. H.; Cyrier, M.; Fleming, G. R. J. Chem. Phys. 2001, 114, 8008.

(49) Montrose, C. J.; Bucaro, J.; Mashall-Coakley, J.; Litowitz, T. A J. Chem. Phys. 1974, 60, 5025.

(50) Mason, P. R.; Hasted, J. B.; Moore, L. Adv. Mol. Relax. Proc. 1974 $6,217$.

(51) Winkler, K.; Lindner, J.; Bürsing, H.; Vöhringer, P. J. Chem. Phvs. $2000,113,4674$.

(52) Winkler, K.; Lindner, J.; Vöhringer, P. Phvs. Chem. Chem. Phys. 2002, 4, 2144.

(53) Foggi, P.; Bellini, M.; Kien, D. P.; Vercuque, I.; Righini, R. J. Phys. Chem. 1997, 101, 7029.

(54) Rønne, C.; Åstrand, P.-O.; Keiding, S. R. Phys. Rev. Lett. 1999, 82,2888 . 
(55) Rønne, C.; Thrane, L.; Astrand, P. O.; Wallqvist, A.; Mikkelsen,

K. V.; Keiding, S. R. J. Chem. Phys. 1997, 107, 5319.

(56) Woutersen, S.; Bakker, H. J. Nature 1999, 402, 507.

(57) Lang, M. J.; Jordanides, X. J.; Song, X.; Fleming, G. R. J. Chem. Phys. 1999, 110, 5884

(58) Schrader, W.; Kaatze, U. J. Phys. Chem. B 2001, 105, 6266.

(59) Marsh, D. Handbook of Lipid Bilayers; CRC Press: Boca Raton, FL, 1990.

(60) Heimburg, T. Biophys. J. 2000, 78, 1154.

(61) Spink, C. H.; Clouser, D.; O’Neil, J. Biochim. Biophys. Acta 1994, 1191, 164

(62) Spink, C. H.; Yeager, M. D.; Feigenson, G. W. Biochim. Biophvs. Acta 1990, 1023, 25.

(63) Klausner, R. D.; Wolf, D. E. Biochemistry 1980, 19, 6199.
(64) Rappolt, M.; Pabst, G.; Rapp, G.; Kriechbaum, M.; Amenitsch, H.; Krenn, C.; Bernstorff, S.; Laggner, P. Eur. Biophys. J. 2000, 29, 125 .

(65) Le Bihan, T.; Pezolet, M. Chem. Phys. Lipids 1998, 94, 13.

(66) Channareddy, S.; Janes, N. Biophvs. J. 1999, 77, 2046.

(67) Westlund, P. O. J. Phys. Chem. B 2000, 104, 6059.

(68) Zhou, Z.; Sayer, B. G.; Hughes, D. W.; Stark, R.; Epand, R. M. Biophys. Chem. 1999, 76, 387.

(69) Bechinger, B.; Seelig, J. Chem. Phys. Lipids 1991, 58, 1.

(70) Ulrich, A. S.; Watts, A. Biophys. Chem. 1994, 49, 39.

(71) Ulrich, A. S.; Watts, A. Biophys. J. 1994, 66, 1441.

(72) Wassall, S. R. Biophys. J. 1996, 71, 2724.

(73) Volke, F.; Eisenblätter, S.; Galle, J.; Klose, G. Chem. Phys. Lipids 1994, 70, 121. 